\title{
Forward Link Capacity in Multi-service DS-CDMA Systems
}

\author{
Lei Zhuge and Victor O. K. Li
}

\begin{abstract}
In this paper the capacity of the forward link in multiservice DS-CDMA systems is analyzed. Capacity constraints are derived with respect to the number of users in each service class. Shadowing, hard/soft handoffs, and data rate variations are considered in the analysis. Solutions of the capacity constraints are used to compare the forward link capacity with hard and soft handoffs. The accuracy of the solutions is verified by simulations.
\end{abstract}

\section{INTRODUCTION}

The future wideband DS-CDMA (direct sequence code division multiple access) systems are expected to provide various types of applications besides the traditional voice service. These applications include transmissions of text messages, images, web pages, audio and video streams, to name a few. Several system level problems, such as admission control, quality of service (QoS) guarantee, burst scheduling, etc., arise in these wireless multimedia systems. A fundamental issue behind these problems, however, is the system capacity analysis.

The forward link (or downlink) in DS-CDMA, due to its synchronism and orthogonality, has smaller intra-cell interference, higher power control efficiency, and lower error rate than the reverse link [1]. In spite of all these advantages, with the adoption of coherent and multi-user detection techniques on the reverse link and the asymmetric property of network traffic, the forward link may become the bottleneck of the system [2]. Simulations are performed to show the forward link capacity under the current or proposed system configurations [2], [3], [4]. Unfortunately, it is inconvenient to apply these simulation results to real-time capacity control, and such simulations do not consider the different traffic types in a multi-service system.

Another related issue is soft handoff on the forward link. Unlike the reverse link where the user's signal is received by the neighboring base stations whether or not it is in soft handoff, additional transmissions from the neighboring base stations are required for forward-link soft handoff, and thus more power is consumed and more interference is produced in the neighboring cells. Therefore, whether soft handoff can really increase the forward link capacity or not is arguable [1].

In this paper we analyze the forward link capacity, and derive the capacity constraints with respect to the number of users

L. Zhuge is with the Communication Sciences Institute, Department of Electrical Engineering - Systems, University of Southern California, Los Angeles, CA 90089-2565, USA (e-mail: lzhuge@usc.edu).

V. O. K. Li is with the Department of Electrical and Electronic Engineering, The University of Hong Kong, Pokfulam, Hong Kong, China (e-mail: vli@eee.hku.hk).

This research is supported in part by the Research Grant Council, Hong Kong, under Grant No. HKU 7047/00E. in each service class. Gaussian and lognormal approximations are used to make the analysis tractable. Shadowing, hard/soft handoffs, and data rate variations are taken into account in the analysis. Simulation results show that the lognormal approximation produces reasonably accurate solutions at the normal operation values of outage probabilities. In addition, comparison of the solutions between hard and soft handoff indicates that soft handoff can provide considerable gain on the forward link capacity, which agrees with the published simulation data. This work is complementary to our previous analysis on the reverse link capacity in multi-service DS-CDMA systems [8].

The forward link capacity analysis is performed in Section II. Numerical results are given in Section III before the concluding section.

\section{ForWARD LinK CAPACITY ESTIMATION}

\section{A. The Outage Constraint}

In either forward or reverse link of a DS-CDMA system, the capacity is limited by the total interference in the system. The system interference level must be maintained below a certain threshold so that the users' communication quality can be guaranteed. Usually the parameter bit-energy-to-interferencedensity ratio (BIR) is used as the quality of service $(\mathrm{QoS})$ index. The BIR of a user $i$ is calculated, by definition, as [1]

$$
\gamma_{i} \triangleq \frac{E_{b, i}}{I_{0, i}}=\frac{S_{i}}{I_{i}} \frac{W}{R_{i}}
$$

where $S_{i}, E_{b, i}$, and $R_{i}$ are the received signal power, bit energy, and data rate of the user $\left(E_{b, i}=S_{i} / R_{i}\right), I_{i}$ and $I_{0, i}$ are the total interference power and its density received by the user $\left(I_{0, i}=\right.$ $\left.I_{i} / W\right)$, and $W$ is the system bandwidth, respectively.

Let $S_{j i}$ be the total power received by user $i$ from the base station in cell $j$, where $S_{0 i}$ is the total power from the user's own base station (in cell 0 ). Let $\phi_{i}$ be the fraction of base station power for the transmission to user $i$. The interference power $I_{i}$ consists of intra-cell and other-cell interference. The intracell interference is caused by the partial loss of orthogonality among the user codes due to the multipath effect, and is equal to $\left(1-f_{o}\right) S_{0 i}$ where $f_{o}$ is the orthogonality factor [2], [3]. The other-cell interference is the sum of the power received from all the neighboring base stations. Therefore,

$$
\gamma_{i}=\frac{\phi_{i} S_{0 i}}{\left(1-f_{o}\right) S_{0 i}+\sum_{j=1}^{M} S_{j i}} \frac{W}{R_{i}}
$$


where $M$ is the number of neighboring cells under consideration. Thermal noise is ignored in (2) ${ }^{1}$.

Assume homogeneous traffic distribution, i. e. the types and amount of traffic are the same in all the cells, and the users are uniformly distributed in the cell. Assume further that the total transmission power from each base station is equal. The path loss model with shadowing is well known and given by [1]

$$
\alpha(r)=r^{m} \epsilon
$$

where $r$ is the distance, $m$ is the path loss exponent, and $\epsilon$ is a lognormal random variable with parameters $\left(0, \sigma_{\epsilon}^{2}\right)$. Since the received power is inversely proportional to the path loss, we have, from (2),

$$
\phi_{i}=\frac{R_{i} \gamma_{i}}{W}\left(1-f_{o}+\sum_{j=1}^{M} \frac{S_{j i}}{S_{0 i}}\right)=\frac{R_{i} \gamma_{i} l_{i}}{W}
$$

where

$$
l_{i} \triangleq 1-f_{o}+\sum_{j=1}^{M} \frac{S_{j i}}{S_{0 i}}=1-f_{o}+\sum_{j=1}^{M}\left(\frac{r_{0 i}}{r_{j i}}\right)^{m} \frac{\epsilon_{0 i}}{\epsilon_{j i}}
$$

and we call $l_{i}$ the modified path loss ratio of the user.

Given physical channel characteristics and cell device configurations, $\gamma_{i}$ is a known value. Hence $\phi_{i}$ varies with the data rate $R_{i}$ and the modified path loss ratio $l_{i}$. In the usual cases when the $\operatorname{BIR}\left(\gamma_{i}\right)$ is guaranteed for all the users in cell 0 , if a fraction $\beta$ of the base station power is dedicated to common control channels (pilot, paging, synchronization, etc.), the sum of $\phi_{i}$ of all users in the cell should not exceed $1-\beta$. Therefore, for normal operation of the system, there is a limit on the outage probability, or

$$
\operatorname{Pr}\left\{\sum_{i=1}^{N} \phi_{i}>1-\beta\right\}<\delta
$$

is required, where $N$ is the total number of users in the cell.

\section{B. Approximations and Capacity Constraints}

In a multi-service system, let $L$ be the total number of service classes, and $N_{k}$ the number of class- $k$ users in the cell ( $k=$ $1, \ldots, L)$. The outage constraint (6) is rewritten as

$$
\operatorname{Pr}\left\{\Phi \triangleq \sum_{k=1}^{L} \sum_{i=1}^{N_{k}} \phi_{k i}>1-\beta\right\}<\delta
$$

where

$$
\phi_{k i}=\frac{R_{k i} \gamma_{k} l_{k i}}{W} ; \quad l_{k i}=1-f_{o}+\sum_{j=1}^{M}\left(\frac{r_{0 k i}}{r_{j k i}}\right)^{m} \frac{\epsilon_{0 k i}}{\epsilon_{j k i}} .
$$

\footnotetext{
${ }^{1}$ Equation (2) assumes that the signal power for a single user is small compared with the total interference power in a cell.
}

Our aim is to obtain a relationship among $N_{k}(k=1, \ldots, L)$ from the constraint (7). The Gaussian approximation has been used on the reverse link outage analysis (see, for example, In [1], [5]). It is shown that the solutions under the Gaussian approximation are reasonably close to the simulation results, although the central limit theorem is not strictly applicable. In our case the capacity constraint obtained by the Gaussian approximation is

$$
m_{\Phi}+T_{g} \sqrt{v_{\Phi}}<1-\beta \quad \text { (Gaussian approx.) }
$$

where $T_{g}$ defines the tail probability $\delta$ of a standard Gaussian random variable, or $\int_{T_{g}}^{\infty} \frac{1}{\sqrt{2 \pi}} e^{-x^{2} / 2} d x=\delta$, and

$$
\begin{aligned}
& m_{\Phi}=\sum_{k=1}^{L} N_{k} \frac{\gamma_{k}}{W} m_{R_{k}} m_{l_{k}} \\
& v_{\Phi}=\sum_{k=1}^{L} N_{k}\left(\frac{\gamma_{k}}{W}\right)^{2}\left(v_{R_{k}} v_{l_{k}}+m_{R_{k}}^{2} v_{l_{k}}+m_{l_{k}}^{2} v_{R_{k}}\right)
\end{aligned}
$$

where $m_{x}$ and $v_{x}$ denote the mean and variance of the random variable $x$, respectively. The moments of $R_{k}$ and $l_{k}$ are discussed in the following sub-sections.

We have found by simulations, however, that the Gaussian approximation tends to overestimate the cell capacity in a multiservice environment. The error is especially obvious when the traffic is bursty. The major reason, we think, is that the lognormal component $\epsilon_{0 k i} / \epsilon_{j k i}$ in $\Phi$ (see (7) and (8)) has a "longer tail" in its density function than a Gaussian random variable. ${ }^{2}$ Traffic burstiness increases further the variance of $\Phi$, resulting in underestimating the tail probability in the Gaussian approximation.

It is therefore reasonable to approximate $\Phi$ by a lognormal random variable (as we did in [8] for the reverse link) which is scaled from the shadowing variable $\epsilon$ and has the same mean and variance as $\Phi$. That is,

$$
\Phi \approx m_{\Phi}+\left(\epsilon-m_{\epsilon}\right) \sqrt{\frac{v_{\Phi}}{v_{\epsilon}}}
$$

Then the capacity constraint under this lognormal approximation can be proved to be

$$
m_{\Phi}+\left(T_{l n}-m_{\epsilon}\right) \sqrt{\frac{v_{\Phi}}{v_{\epsilon}}}<1-\beta \quad \text { (Lognormal approx.) }
$$

where $T_{l n}$ is determined by $\int_{T_{l n} n}^{\infty} \frac{10}{\ln 10 \sqrt{2 \pi} \sigma_{\epsilon} x} e^{-\frac{\left(10 \log _{10} x\right)^{2}}{2 \sigma_{\epsilon}^{2}}} d x=$ $\delta$.

Our simulations show that the lognormal approximation is more accurate than the Gaussian approximation in the region around $\delta=0.01$ (the region corresponding to most DS-CDMA system designs).

\footnotetext{
${ }^{2}$ The division of two shadowing variables is a lognormal random variable with the same parameters (i. e. another shadowing variable), if the two components in the shadowing (with respect to the user and the base station, respectively) are assumed equal [1].
} 


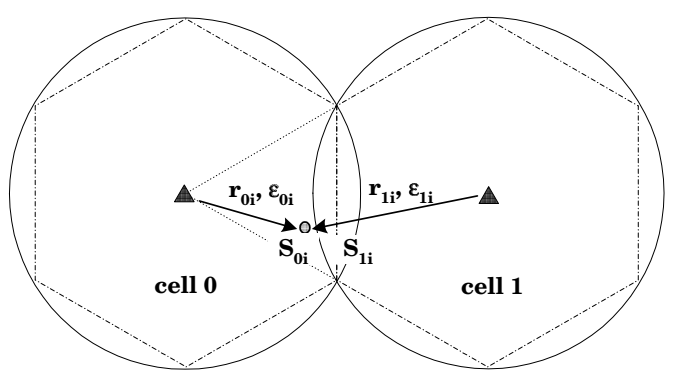

Fig. 1. Handoff between two cells

\section{Handoff Considerations}

Handoff can occur even if a user does not cross the cell boundary, when the received pilot from a neighboring base station is strong compared with the pilot from the local cell. (8) has to be modified to accommodate handoff cases.

\section{Hard Handoff}

In the hysteresis model [6], [7], hard handoff does not occur until the pilot from the neighboring cell (say, cell 1) is stronger than that from the local cell (cell 0 ) by a margin $\Delta_{d B}$. This condition is equivalent to the path loss from cell 0 being $\Delta_{d B}$ higher than that from cell 1. After handoff the user is powercontrolled by cell 1 , and the fraction of power in base station 1 consumed by the signal to the user is (for convenience we omit the subscript $k$ indicating service class)

$$
\phi_{i}^{\prime}=\frac{R_{i} \gamma_{i}}{W}\left(1-f_{o}+\sum_{j=0, j \neq 1}^{M} \frac{S_{j i}}{S_{1 i}}\right)
$$

Under the assumption of uniform user distribution, due to symmetry, the scenario of a user in cell 0 power-controlled by cell 1 is completely analogous to that of a user in cell 1 powercontrolled by cell 0 . Hence, we can take $\phi_{i}^{\prime}$ as the fraction of power in base station 0 consumed by a user in cell 1 but powercontrolled by cell 0 . Therefore, we redefine the modified path loss ratio as (see Fig. 1 for reference)

$l_{i}=\left\{\begin{array}{l}1-f_{o}+\sum_{j=1}^{M}\left(\frac{r_{0 i}}{r_{j i}}\right)^{m} \frac{\epsilon_{0 i}}{\epsilon_{j i}} \text { when }\left(\frac{r_{0 i}}{r_{1 i}}\right)^{m} \frac{\epsilon_{0 i}}{\epsilon_{1 i}}<\Delta \\ 1-f_{o}+\sum_{j=0, j \neq 1}^{M}\left(\frac{r_{1 i}}{r_{j i}}\right)^{m} \frac{\epsilon_{1 i}}{\epsilon_{j i}} \text { otherwise }\end{array}\right.$

so that $\phi_{i}=\frac{R_{i} \gamma_{i} l_{i}}{W}$ is still valid and the capacity constraints derived in the last sub-section still hold.

\section{Soft Handoff}

Consider soft handoff between the user's two nearest base stations in cell 0 and cell 1 . User $i$ in cell 0 enters soft handoff when the received strength of the pilot from cell $1\left(p_{1 i}\right)$ is greater than that from cell $0\left(p_{0 i}\right)$ minus $\Delta_{d B}$. The soft handoff status remains until $p_{1 i}$ exceeds $p_{0 i}$ plus $\Delta_{d B}$. Assume that in the soft handoff phase the two base stations transmit to the user with the
TABLE I

MeAn AND VARIANCE OF MOdified PATH Loss Ratio $\left(m=4, \Delta=6 \mathrm{~dB}, f_{o}=0.7\right)$

\begin{tabular}{ccccc}
\hline $\begin{array}{c}\text { Shadowing } \\
\text { dev. } \sigma_{\epsilon}\end{array}$ & \multicolumn{2}{c}{ Hard handoff } & \multicolumn{2}{c}{ Soft handoff (2-way) } \\
mean $m_{l}$ & var. $v_{l}$ & mean $m_{l}$ & var. $v_{l}$ \\
\hline $2 \mathrm{~dB}$ & 1.065 & 0.877 & 1.060 & 0.547 \\
$4 \mathrm{~dB}$ & 1.083 & 1.002 & 1.061 & 0.576 \\
$6 \mathrm{~dB}$ & 1.123 & 1.293 & 1.075 & 0.702 \\
$8 \mathrm{~dB}$ & 1.265 & 2.898 & 1.181 & 1.740 \\
\hline
\end{tabular}

same fraction $\phi_{i}$ of the base station power. The user, as a result, receives a total signal power of $S_{0 i}+S_{1 i}$, and at the same time consumes a total fraction $2 \phi_{i}$ of the power in the two base stations. The modified path loss ratio $l_{i}$ can then be derived as

$$
\begin{gathered}
l_{i}=2\left\{1-f_{o}+\left[\sum_{j=1}^{M}\left(\frac{r_{0 i}}{r_{j i}}\right)^{m} \frac{\epsilon_{0 i}}{\epsilon_{j i}}\right] /\left[1+\left(\frac{r_{0 i}}{r_{1 i}}\right)^{m} \frac{\epsilon_{0 i}}{\epsilon_{1 i}}\right]\right\} \\
\text { when } \frac{1}{\Delta}<\left(\frac{r_{0 i}}{r_{1 i}}\right)^{m} \frac{\epsilon_{0 i}}{\epsilon_{1 i}}<\Delta
\end{gathered}
$$

When the user is not in the handoff region (i. e. $\left(r_{0 i} / r_{1 i}\right)^{m} \epsilon_{0 i} / \epsilon_{1 i}>\Delta$ or $\left.\left(r_{0 i} / r_{1 i}\right)^{m} \epsilon_{0 i} / \epsilon_{1 i}<1 / \Delta\right)$, the expressions for $l_{i}$ have the same format as (14).

Soft handoff involving more than two base stations can be tackled in a similar way.

The Moments of $l_{i}$

The distribution of $l_{i}$ defined by (14) and (15) can not be obtained analytically. However, we can use Monte Carlo simulation to study its characteristics. The mean and variance of $l_{i}$ with different shadowing deviations, obtained by Monte Carlo simulation using a layout of two-tier (totally 18) neighboring cells and circular cells with 60-degree overlapping (Fig. 1) ${ }^{3}$, are listed in Table I. As expected, soft handoff can reduce both mean and variance of the modified path loss ratio (and thus reduce the other cell interference).

\section{The Traffic Model}

In order to characterize the data rate variations, we use the two-state Markov model for traffic streams. Assume that any time each transmission is in either of the two states, $a$ and $b$, and in each state it generates traffic with a fixed data rate, $R_{a}$ or $R_{b}$, respectively. The holding time at each state is exponential. Under this model the traffic data rate at any given time is a random variable taking two values

$$
R=\left\{\begin{array}{lll}
R_{a} & \text { with prob. } & \rho \\
R_{b} & \text { with prob. } & 1-\rho
\end{array}\right.
$$

where $\rho$ is usually called the activity factor of the source if $R_{b}$ $=0 . R$ has mean $m_{R}$ and variance $v_{R}$ given by

$$
m_{R}=\rho R_{a}+\left(1-p R_{b}, \quad v_{R}=\rho(1-\not)\left(R_{a}-R_{b}\right)^{2}\right.
$$

${ }^{3}$ We use overlapping circular cells because they are more realistic than hexagonal ones. 
TABLE II

EXAmPle SySTEM AND TRAFFiC PARAMETERS

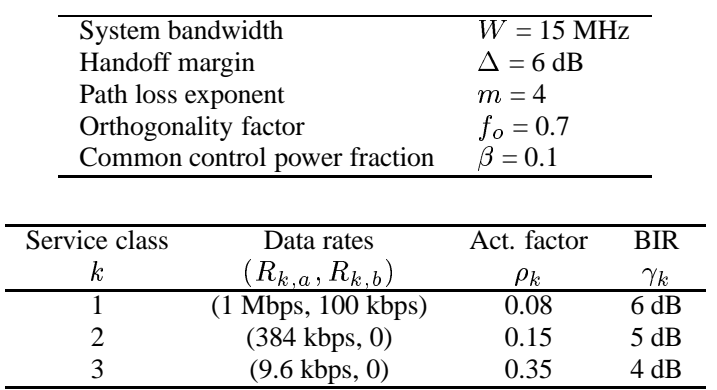

This model can be applied to a wide range of traffic types including voice and bursty data. Generally any traffic model can be used in our capacity analysis, since we only need the mean and variance of the data rate. The major concern, however, is how the traffic variations influence the accuracy of the approximations under which the capacity constraints are derived.

\section{E. Virtual Bandwidth}

To quantitatively determine the system capacity in a multiservice environment, we use the virtual bandwidth concept introduced in our previous work [8], [9] for reverse link analysis as the capacity metric ${ }^{4}$. For the forward link we redefine system virtual bandwidth as

$$
C=\sum_{k=1}^{L} N_{k} m_{R_{k}} \gamma_{k}\left(1-f_{o}\right)
$$

The factor $1-f_{o}$ is used to scale the value of $C$ down to the level of the system bandwidth.

$C$ may be thought of as the average system bandwidth "effectively utilized" by all users in the system. The maximum value of $C$ is $W(1-\not \beta$ for the forward link of an isolated cell (i. e. if we do not consider other-cell interference, see (4) and (6) for reference). In case all the service classes has the same $\operatorname{BIR}\left(\gamma_{k}\right)$ requirement, the system virtual bandwidth $C$ is proportional to the average aggregate data rate $\sum_{k=1}^{L} N_{k} m_{R_{k}}$.

\section{NUMERICAL RESUlTS}

\section{A. Solutions and Approximation Errors}

We consider a system with three diverse traffic types (service classes) as listed in the second part of Table II. Classes 1 and 2 are high-speed bursty traffic (class 1 traffic is similar to an MPEG stream), while class 3 is voice-like traffic. Table III shows a set of solutions on the number of users $N_{k}(k=1,2,3)$ under Gaussian and lognormal approximations ((9) and (12)), respectively.

Based on these solutions we perform simulation to "measure" the outage probabilities and compare them with the target values, so that the errors of the Gaussian and lognormal approximations can be examined. The simulation is performed on the 19

\footnotetext{
${ }^{4}$ We notice that a similar concept was also proposed in [10].
}

TABLE III

A Set of Solutions of the Capacity Constraints ( $\sigma_{\epsilon}=6 \mathrm{~dB}$, hard handoff)

\begin{tabular}{lcccccc}
\hline Target & \multicolumn{3}{c}{ Gaussian approx. } & \multicolumn{3}{c}{ Lognormal approx. } \\
outage $\delta$ & $N_{1}$ & $N_{2}$ & $N_{3}$ & $N_{1}$ & $N_{2}$ & $N_{3}$ \\
\hline 0.001 & 2 & 7 & 87 & 0 & 3 & 160 \\
0.002 & 2 & 9 & 66 & 0 & 5 & 87 \\
0.005 & 2 & 12 & 45 & 0 & 9 & 35 \\
0.01 & 3 & 9 & 71 & 1 & 8 & 45 \\
0.02 & 3 & 12 & 70 & 2 & 9 & 64 \\
0.05 & 4 & 13 & 62 & 4 & 12 & 62 \\
0.1 & 4 & 19 & 52 & 5 & 21 & 34 \\
\hline
\end{tabular}

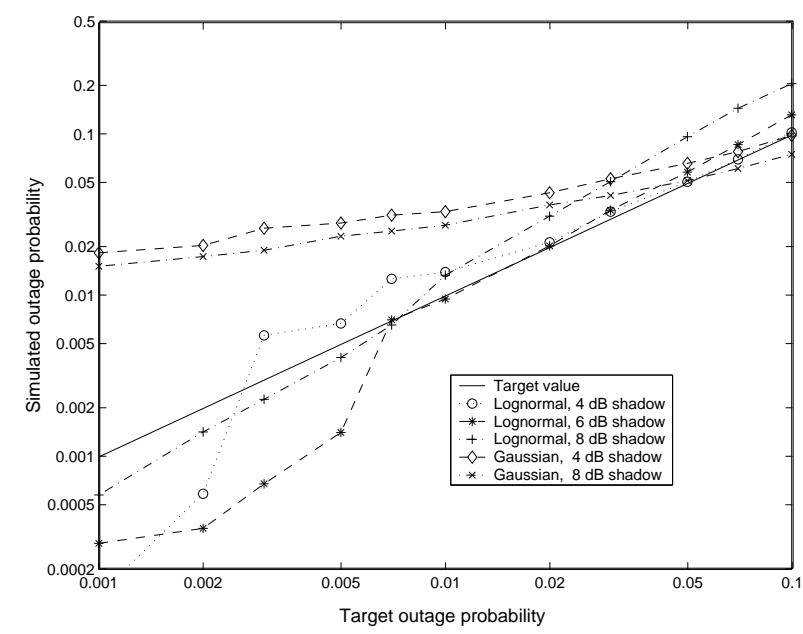

Fig. 2. Simulated outage probabilities by solutions with lognormal approximation (hard handoff)

overlapping circular cell layout described in Section II-C. For each user, 100,000 random samples are generated for its location, data rate, and shadowing, respectively, according to their stochastic models. The power fraction $\phi_{i}$ is then calculated by (4) and (14) for hard handoff or (15) for soft handoff, and added together to form the samples of $\Phi$ (7). Then the frequency of the samples exceeding $1-\beta$ is calculated as the simulated outage probability. Such experiments are repeated $10-20$ times for each solution, and the average simulated outage probabilities are shown in Fig. 2.

It is seen from Fig. 2 that the simulated results from the lognormal approximation generally follows the slope of the change of target outage probabilities, so long as the target value is not too small (below 0.007). The Gaussian approximation, on the other hand, tends to allow more traffic than acceptable in most of the outage probability region. The lognormal method outperforms the Gaussian one especially in the region around $\delta=0.01$ which is the operating region in most real DS-CDMA systems. We have noticed the sudden drop of the system capacity using the lognormal method at small outage probabilities and are seeking the reason for it. One of the possibilities is that when $\delta$ is small, few high-speed bursty transmissions are allowed, and the traffic variation is smaller than what the lognormal approximation expects. 


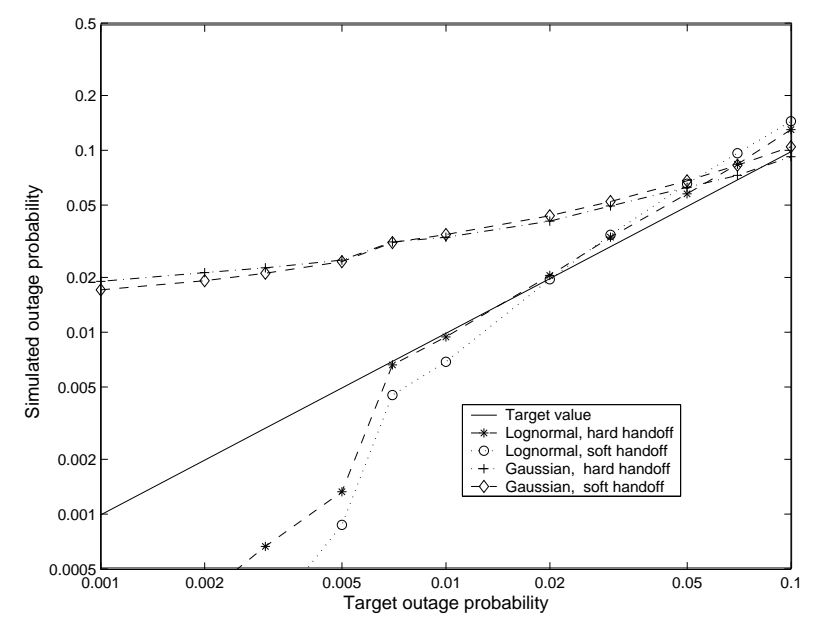

Fig. 3. Simulated outage probabilities with hard and soft handoffs $\left(\sigma_{\epsilon}=6 \mathrm{~dB}\right)$

TABLE IV

CAPACITY COMPARISON BETWEEN HARD AND SOFT HANDOFF ( $\sigma_{\epsilon}=6 \mathrm{~dB}$, Lognormal approximation)

\begin{tabular}{ccccccc}
\hline Target & \multirow{2}{*}{$N_{1}$} & $N_{2}$ & \multicolumn{2}{c}{$N_{3}$} & \multicolumn{2}{c}{ Virtual b.w. $C(\mathrm{MHz})$} \\
outage $\delta$ & & & hard h.o. & soft h.o. & hard h.o. & soft h.o. \\
\hline 0.007 & 1 & 6 & 12 & 222 & 0.564 & 1.095 \\
0.01 & 1 & 8 & 45 & 245 & 0.757 & 1.263 \\
0.02 & 2 & 9 & 64 & 253 & 1.065 & 1.543 \\
0.03 & 3 & 9 & 67 & 248 & 1.278 & 1.736 \\
0.05 & 4 & 12 & 62 & 226 & 1.634 & 2.050 \\
0.07 & 4 & 18 & 51 & 200 & 1.934 & 2.312 \\
0.1 & 5 & 21 & 34 & 166 & 2.261 & 2.595 \\
\hline
\end{tabular}

\section{B. The Effect of Soft Handoff}

To facilitate the capacity comparison between hard and soft handoff, we choose the same number of class 1 and 2 users $\left(N_{1}\right.$ and $N_{2}$ ) for hard and soft handoffs, and calculate $N_{3}$ from the capacity constraint. The results under the lognormal approximation are listed in Table IV. We use the values for $\delta \geq 0.007$ and only those with lognormal approximation because the capacity estimate under these conditions is very close to the simulation result as shown in Fig. 3. We choose the shadowing deviation $\sigma_{\epsilon}=6 \mathrm{~dB}$ as this is a typical value in the micro-cell environment [2].

Table IV indicates that soft handoff obviously increases the forward link capacity as it allows much more class 3 users than in hard handoff for given $N_{1}$ and $N_{2}$ values. To clearly specify the capacity gain achieved by soft handoff over hard handoff, the system virtual bandwidths are calculated by (18) for both soft and hard handoffs. It is shown that the system virtual bandwidth offered by soft handoff is $15 \%-94 \%$ higher than that by hard handoff. This agrees with the simulation result in [3] on the CODIT testbed where the spectrum efficiency (in Kbps per $\mathrm{MHz}$ ) is used for system capacity.

However, we need to be cautious about this capacity gain provided by soft handoff, because it is obtained under certain assumptions. In real systems we can not guarantee that the two base stations involved in the soft handoff always transmit to the user with equal power, and the variation in the transmitting power may change the system capacity. It is also possible that the signal power from the two base stations can not be fully captured by the mobile user due to limited RAKE fingers in the receiver device [3]. In addition, unless the traffic distribution is completely homogeneous in all the cells, we have to consider, during the soft handoff, the heavy interference added to the neighboring cells when a high-speed bursty data user stays close to the cell boundary. Due to the last concern, it is suggested that a stationary and high-speed packet data user communicate with only one base station for downlink transmissions [2].

\section{Discussions AND CONCLUSIONS}

The following issues may also be considered in our forward link capacity analysis.

- Soft handoff with more base stations only affects the modified path loss ratio with different handoff regions.

- Cell sectorization causes the user to receive signals from less base stations. It also affects the modified path loss ratio.

- Constraints on the Erlang capacity of each service class can be derived similarly given the user arrival models.

- Data retransmission is approximately equivalent to a slight increase on the data rate or data arrival rate.

The capacity constraints derived in this paper are easy to formulate and solve. Solutions by the lognormal approximation are shown to have reasonably good accuracy in the normal range of outage probabilities. However, the behavior of the lognormal approximation must be studied further to examine its error range under different variable factors, before it can be applied to real system problems.

\section{REFERENCES}

[1] A. J. Viterbi, CDMA: Principles of spread spectrum communication, Addison-Wesley Pub., 1995.

[2] T. Ojanperä and R. Prasad, Wideband CDMA for the third generation mobile communications, Artech House pub., 1998.

[3] S. Hämäläinen, H. Holma, A. Toskala, and M. Laukkanen, "Analysis of CDMA downlink capacity enhancements," Proc. IEEE PIMRC'97, Sep. 1997, pp. 241-245.

[4] R. Walton, M. Wallace, and S. Howard, "CDMA downlink performance issues,” Proc. IEEE PIMRC'98, Sep. 1998, pp. 308-312.

[5] J. S. Evans and D. Everitt, "On the teletraffic capacity of CDMA cellular networks," IEEE Trans. Vehicul. Tech., vol. 48, no. 1, Jan. 1999, pp. 153165.

[6] V. O. K. Li and X. Qiu, "Personal communication systems (PCS)," Proc. IEEE, vol. 83, no. 1, Sep. 1995, pp. 1210-1243.

[7] K. M. Rege, S. Nanda, C. F. Weaver, and W.-C. Peng, "Analysis of fade margins for soft and hard handoffs," Proc. IEEE PIMRC'95, Sep. 1995, pp. 829-835.

[8] L. Zhuge and V. O. K. Li, "Interference estimation for admission control in multi-service DS-CDMA cellular systems," Proc. IEEE GLOBECOM'O0, Nov. 2000, pp. 1509-1514.

[9] L. Zhuge and V. O. K. Li, "Reverse-link capacity of multiband overlaid DS-CDMA," accepted for ACM/Baltzer Mobile Net. Appl., Mar. 2002.

[10] D. Shen and C. Ji, "Admission control of multimedia traffic for third generation CDMA network," Proc. IEEE INFOCOM'O0, Mar. 2000, pp. 1077 1086 